\title{
Biebricher, Thomas, und Ralf Ptak (2020): Soziale Marktwirtschaft und Ordoliberalismus zur Einführung
}

\author{
Hamburg: Junius. 256 Seiten. 15,90€
}

\section{Felix Schilk iD}

Online publiziert: 26. Oktober 2020

(C) Der/die Autor(en) 2020

In kaum einem Begriff verdichtet sich das Selbstbild der Bundesrepublik derart wie in der Idee der ,Sozialen Marktwirtschaft““. Als staatstragendes Narrativ und identitätsstiftender Mythos synchronisiert der Begriff über Lagergrenzen hinweg die politisch-mediale Kommunikation und ist Ausgangspunkt zahlreicher Legenden, die Ulrike Herrmann in „Deutschland, ein Wirtschaftsmärchen“ jüngst einer kritischen Prüfung unterzogen hat. Als Objekt von diskursiven Deutungskämpfen wird er von unterschiedlichsten Akteuren wie Parteien, Gewerkschaften, Arbeitgeberverbänden und Thinktanks strategisch angeeignet und umgedeutet. Eine wissenschaftliche Auseinandersetzung mit dem Konzept der Sozialen Marktwirtschaft steht deshalb vor zwei Problemen: Einerseits muss sie die normative Aufladung des Begriffes berücksichtigen und andererseits die Grenzen des Konzepts als analytische Kategorie reflektieren. Der im Hamburger Junius-Verlag erschienene Einführungsband des Politikwissenschaftlers Thomas Biebricher und des Volkswirts Ralf Ptak nimmt diese Problematik zum Ausgangspunkt, um die Soziale Marktwirtschaft in ihren historischen Debattenkontext einzubetten und als Mythos zu dekonstruieren. Ziel des Buches ist nicht weniger als eine ,alternative Deutung der wirtschaftlichen Nachkriegsgeschichte" (S. 9).

Beide Autoren sind skeptisch, was die Tragfähigkeit des Begriffs als Analysekategorie betrifft und weisen immer wieder darauf hin, dass ein erkenntnisleitender Zugriff die Unterscheidung von verschiedenen Untersuchungsebenen voraussetze. Da die Versuche, die Soziale Marktwirtschaft als „,ein fest umrissenes, gar systematisch wirtschaftswissenschaftliches Theoriegebäude darzustellen, in einer mehr oder weniger interessengeleiteten Konstruktion enden“ (S. 125), werden im Buch vier Zugänge abgesteckt. Die Soziale Marktwirtschaft sei einmal eine „Strategie

F. Schilk $(\square)$

Institut für Soziologie, Technische Universität Dresden, Dresden, Deutschland

E-Mail: felix.schilk@tu-dresden.de 
marktkonformer wirtschaftspolitischer Interventionen“ (S. 17), zweitens ein sowohl wirtschafts- als auch gesellschaftspolitisches Konzept, drittens die ,real existierende Soziale Marktwirtschaft" (S. 18), wie sie als Wirtschafts- und Sozialpolitik in der frühen Bundesrepublik praktiziert wurde, und viertens das bundesrepublikanische Narrativ des wirtschaftlichen Aufschwungs in der Nachkriegszeit.

Diese „historisch, konzeptionell, realwirtschaftlich“ (S. 78) orientierten Perspektiven werden von Biebricher und Ptak durch die Leitunterscheidung von Sozialer Marktwirtschaft und Ordoliberalismus geordnet und auf verschiedenen Handlungsebenen situiert, „die im Kern nicht vergleichbar sind“ (S. 23). So wird der Ordoliberalismus als dogmatische Theoriekonzeption und deutsche Spielart des Neoliberalismus verstanden, während die Soziale Marktwirtschaft als pragmatische und flexible Implementierungsstrategie untersucht wird, die einem zeitlichen Wandel unterliege und eine ,enorme programmatische Varianz“ (S. 122) aufweise. Dabei befinde sich die Soziale Marktwirtschaft stets ,in einem Spannungsverhältnis zwischen politisch-ideologischer Intention und ordoliberalem Leitbild“ (S. 107), schreibt Ptak und kritisiert übermäßige Differenzierungen durch komparative Ansätze, die etwa „die Funktion der verschiedenen Spielarten des Neoliberalismus in unterschiedlichen Zeiten und unter verschiedenen sozioökonomischen Rahmenbedingungen vor dem Hintergrund spezifischer kultureller Settings“" (S. 21) verkennen. Mit ihrem Fokus auf der Funktion der Sozialen Marktwirtschaft folgen die beiden Autoren dem Ansatz von Quinn Slobodian, den Neoliberalismus als eine Theorie der Governance zu betrachten, in deren Zentrum ein ,dualistisches Denkgebäude von Marktwirtschaft und gesellschaftlicher Integrationssphäre“ (S. 66) steht und die das Ziel einer „außerökonomischen Integration innerhalb der Marktgesellschaft“ (S. 66) verfolgt.

Der gesellschaftstheoretische Hintergrund des Ordoliberalismus und seine Umsetzung im Rahmen der deutschen Nachkriegsordnung wird in zwei dichten Kapiteln von Ptak anhand zahlreicher Originalquellen eingeführt und von Biebricher in einem weiteren, zeithistorisch angelegten Kapitel bis in die Gegenwart nachverfolgt. Während Ptak das ordoliberale Gesellschaftsbild immanent rekonstruiert, in einer konservativen und antipluralistischen Traditionslinie verortet und immer wieder auf die Dilemmata und Ambivalenzen der ,,paradoxen Mischung aus unerschütterlichem Marktglauben einerseits und reaktionärem Fortschrittspessimismus andererseits“ (S. 59) hinweist, widmet sich Biebricher den westdeutschen Debatten und politischen Reformprojekten von Helmut Kohls „geistig-moralischer Wende“ bis zu Gerhard Schröders „Agenda 2010“. Im Gegensatz zu Ptak greift er dabei kursorisch auf politikwissenschaftliche Klassifikationsschemata wie Esping-Andersens Sozialstaatstypologie oder Hall und Soskices „,Varieties of Capitalism“ zurück und diskutiert pointiert ihre Stärken und Grenzen. Den Abschluss des Buches bildet ein exploratives Kapitel, in dem Biebricher die Transformation des Ordoliberalismus zu einer Ordnungsökonomik untersucht und die Positionierung ordoliberaler Stimmen in der Euro- und Finanzkrise nachzeichnet. Wo die anderen Kapitel klar strukturiert sind und sich an den eingeführten Untersuchungsebenen orientieren, ist dieses Kapitel jedoch erratisch und zum Teil zu sehr Spezialdiskurs, um dem Anspruch einer Einführung gerecht zu werden. Besonders überraschend ist dabei, dass die Entstehung der AfD und ihr Verhältnis zum Ordoliberalismus kaum eine Rolle spielen, 
obwohl der ehemalige Europaabgeordnete und Vorsitzende der Aktionsgemeinschaft Soziale Marktwirtschaft, Joachim Starbatty, mehrfach zitiert wird.

Sieht man von diesen Schwächen ab, dann bietet das Buch ein kompaktes Panorama der Sozialen Marktwirtschaft, das sich auf seinen Gegenstand einlässt, Debattenkontexte distanziert nachzeichnet und seine kritischen Impulse stets an konkreten Quellen entfaltet. Wer eine ideengeschichtliche Verortung des Ordoliberalismus im Raster der politischen Großideologien erwartet, wird jedoch lediglich einige Anregungen erhalten. Eine systematische begriffliche Auseinandersetzung, etwa mit dem Freiheitsbegriff der Ordoliberalen oder dem Konzept des Totalitarismus, hätte die konzeptionelle Perspektive aber mit Sicherheit bereichert. Ärgerlich, aber nicht den Autoren anzulasten, ist die Form der Literaturverweise, die sich als ausführliche Fußnoten am Ende jedes Kapitels befinden. Dass der Verlag auf eine Bibliografie verzichtet, verkompliziert die Sichtung weiterer Literatur auf unnötige Weise.

Funding Open Access funding enabled and organized by Projekt DEAL.

Open Access Dieser Artikel wird unter der Creative Commons Namensnennung 4.0 International Lizenz veröffentlicht, welche die Nutzung, Vervielfältigung, Bearbeitung, Verbreitung und Wiedergabe in jeglichem Medium und Format erlaubt, sofern Sie den/die ursprünglichen Autor(en) und die Quelle ordnungsgemäß nennen, einen Link zur Creative Commons Lizenz beifügen und angeben, ob Änderungen vorgenommen wurden.

Die in diesem Artikel enthaltenen Bilder und sonstiges Drittmaterial unterliegen ebenfalls der genannten Creative Commons Lizenz, sofern sich aus der Abbildungslegende nichts anderes ergibt. Sofern das betreffende Material nicht unter der genannten Creative Commons Lizenz steht und die betreffende Handlung nicht nach gesetzlichen Vorschriften erlaubt ist, ist für die oben aufgeführten Weiterverwendungen des Materials die Einwilligung des jeweiligen Rechteinhabers einzuholen.

Weitere Details zur Lizenz entnehmen Sie bitte der Lizenzinformation auf http://creativecommons.org/ licenses/by/4.0/deed.de. 